\title{
Comunicación
}

\section{Prevalencia de Varroa spp correlacionada al factor productivo y temperamental en el apiario de la Universidad Nacional de Cajamarca, Perú}

\author{
Prevalence of Varroa spp correlated to the productive and temperamental factor in \\ the apiary of the Fundo Tartar Pecuario - Universidad Nacional de Cajamarca, \\ Peru
}

\author{
César A. Murga-Moreno ${ }^{1.4}$, Luis Vargas-Rocha ${ }^{2}$, Miguel Chávez-Farro ${ }^{3}$, \\ Cristian Hobán-Vergara ${ }^{1}$, Pedro Ortiz-Oblitas ${ }^{1}$
}

\section{Resumen}

La varroasis es una enfermedad causada por arácnidos del género Varroa, parásito obligatorio de las abejas y causante de elevada mortalidad. El presente estudio tuvo como objetivo reportar la prevalencia de Varroa spp en el apiario del Fundo Tartar Pecuario de la Universidad Nacional de Cajamarca, Perú y los factores de resistencia como la producción y temperamento de las colmenas y núcleos. El muestreo se realizó en diciembre de 2019. La prevalencia promedio de Varroa spp en los cuatro núcleos muestreados fue de $1.62 \pm 0.61 \%$ y en las tres colmenas de $1.11 \pm 0.5 \%$, siendo de $1.39 \pm 0.41 \%$ para el apiario. Todos los núcleos y colmenas fueron clasificados con buena producción (1 a 2

${ }^{1}$ Laboratorio de Inmunología e Investigación, Facultad de Ciencias Veterinarias, Universidad Nacional de Cajamarca, Cajamarca, Perú

${ }^{2}$ Escuela Académico Profesional de Medicina Veterinaria, Facultad de Ciencias Veterinarias, Universidad Nacional de Cajamarca, Cajamarca, Perú

${ }^{3}$ Departamento Académico de Ciencias Veterinarias, Facultad de Ciencias Veterinarias, Universidad Nacional de Cajamarca, Cajamarca, Perú

${ }^{4}$ E-mail: cmurgam15@unc.edu.pe; https://orcid.org/0000-0002-5879-3694

Recibido: 23 de septiembre de 2020

Aceptado para publicación: 8 de abril de 2021

Publicado: 23 de junio de 2021

CLos autores. Este artículo es publicado por la Rev Inv Vet Perú de la Facultad de Medicina Veterinaria, Universidad Nacional Mayor de San Marcos. Este es un articulo de acceso abierto, distribuido bajo los términos de la licencia Creative Commons Atribución 4.0 Internacional (CC BY 4.0) [https:// creativecommons.org/licenses/by/4.0/deed.es] que permite el uso, distribución y reproducción en cualquier medio, siempre que la obra original sea debidamente citada de su fuente original 
pisos) y en cuanto al temperamento: 1 núcleo tranquilo, 1 agresivo y 2 muy agresivos, mientras que las tres colmenas fueron muy agresivas. Se concluye que la cualidad de ser núcleo o colmena no influye en la producción ni en el temperamento de las abejas. Además, el temperamento o la producción no guardan relación con la presencia de Varroa spp en el apiario.

Palabras clave: Cajamarca, colmenas, núcleos, producción, temperamento, varroasis

\section{Abstract}

Varroasis is a disease caused by arachnids of the genus Varroa, an obligatory parasite of bees and the cause of high mortality. The present study aimed to report the prevalence of Varroa spp in the apiary of the Fundo Tartar Pecuario of the National University of Cajamarca, Peru and resistance factors associated with the production and temperament of the hives and nuclei. The sampling was carried out in December 2019. The average prevalence of Varroa spp in the four nuclei sampled was $1.62 \pm 0.61 \%$ and in the three hives it was $1.11 \pm 0.5 \%$, being $1.39 \pm 0.41 \%$ for the apiary. All the nuclei and hives were classified with good production ( 1 to 2 floors) and in terms of temperament: 1 calm nucleus, 1 aggressive and 2 very aggressive, while the three hives were very aggressive. It is concluded that the quality of being a nucleus or hive does not influence the production or temperament of the bees. Furthermore, temperament or production are not related to the presence of Varroa spp in the apiary.

Key words: Cajamarca, hives, nuclei, production, temperament, varroasis

\section{INTRODUCCIÓN}

El parásito obligado Varroa spp posee un comportamiento forético y representa un problema global en la producción de abejas (Massaccesi, 2002; Espinoza, 2004; Polaino, 2006). Se reconoce que, una población apícola saludable produce $65.5 \%$ más que una población infestada (Arechavaleta y Guzmán, 2000). Así, si la prevalencia de varroasis supera el $10 \%$ en una colmena, la producción de miel empieza a mermar en forma considerable, mientras que una infestación en el $30-40 \%$ de la población ocasiona la destrucción total de la colmena (Franco, 2009). Se ha comprobado también el ingreso de estos ácaros en las colmenas portando enfermedades virales tales como el Virus del Ala Deformada, el Virus de Parálisis Lenta y el Virus Filamentoso, entre otros (Carreck et al., 2010).

La importación de este ácaro hacia América se dio con la introducción de abejas reinas provenientes de Japón a Paraguay en 1971 (Martínez et al., 2011). En el Perú fueron reportados los primeros casos en 1985, principalmente en el valle de Rímac, Lima (Dávila y Ortiz, 1987). En años más recientes, se reporta $80.4 \%$ de prevalencia de esta parasitosis en el Perú, de $93.91 \%$ en la Región Cajamarca, y de $100 \%$ en las regiones de Piura y Ucayali (Mantilla, 2013). 
Se dispone de algunos estudios que describen los factores de resistencia o susceptibilidad de las abejas ante esta acarosis. Por ejemplo, se ha observado que tanto autoacicalamiento como la limpieya entre individuos propician que las varroas se desprendan de las abejas adultas cayendo al piso interior de la colmena (Panziera et al., 2017). El 95\% de los ácaros caen muertos y el resto caen severamente dañados, confiriendo así una mayor resistencia a estas poblaciones (Moncada, 2004; Araneda et al., 2010).

En Ecuador, un trabajo epidemiológico con abejas adultas determinó que la superficie de explotación, aplicación de cera estampada en el apiario, número de cosechas al año, número de marcos de reserva y empleo de suplementos alimenticios no influían significativamente en la presencia de Varroa spp en las colmenas (Arévalo, 2018). Otra investigación en el mismo país concluye que las abejas de temperamento más agresivo (africanizadas), son más resistentes a varroasis que las abejas europeas (Subía, 2013). De la misma manera, un estudio en São Paulo afirma que las abejas africanizadas identifican rápidamente a las crías infestadas con Varroa y las eliminan de las colmenas (Guerra et al., 2000). No obstante, se sugiere también que la propia adaptación poblacional con base genética de las abejas melíferas propicia una mayor resistencia a enfermedades virales y a practicar conductas heredadas de acicalamiento; asimismo, reducen en un $30 \%$ la aptitud reproductiva de las varroas llevándolos en algunos casos a niveles cercanos de infertilidad (Locke et al., 2012).

El objetivo del presente estudio fue determinar la prevalencia de Varroa spp correlacionada al factor productivo y temperamental en núcleos y colmenas del apiario de la Universidad Nacional de Cajamarca.

\section{Materiales y MéTodos}

\section{Colmenas y Núcleos}

El muestreo de las abejas se realizó en el apiario del Fundo Tartar Pecuario de la Universidad Nacional de Cajamarca (UNC), provincia Cajamarca, Región Cajamarca (Perú). En noviembre de 2019 se realizó un inventario del apiario, donde se encontraron tres colmenas y cuatro núcleos. Se les rotuló con pigmentos no tóxicos (témperas Artesco $\left.{ }^{\circledR}\right)$. Además, se las clasificó por el tipo de producción de acuerdo con el número de pisos funcionales (1-2 = Buena; 2-3 = Muy buena; 3-4 = Excelente) y por su temperamento (Tranquilo, Agresivo y Muy agresivo) (Subía, 2013).

\section{Muestras}

El muestreo se realizó a inicios de diciembre de 2019, en horas de la mañana y con abundante brillo solar. Se destapó cada colmena y núcleo para retirar 1 a 2 bastidores que tenían la mayor cantidad de abejas (de preferencia a las cámaras de nido de cría) y se pusieron en posición oblicua sobre la tapa correspondiente a su propia colonia. Luego, se emplearon frascos de boca ancha (con capacidad de $1200 \mathrm{ml}$ ) que tenían en su interior detergente granulado (Marsella ${ }^{\circledR}$ ) disuelto en $800 \mathrm{ml}$ de agua obteniendo una solución final al $2 \%$ por cada muestra. Se deslizó el borde del recipiente de abajo hacia arriba para introducir la mayor cantidad de abejas con una escobilla desabejadora (verificando que la abeja reina no esté en peligro). Se recogió un promedio de 400 abejas adultas de cada colmena y núcleo y se retornó el bastidor a su posición original dentro de la caja. Los frascos fueron tapados y agitados manual y vigorosamente durante 40 segundos. 


\section{Observación y Conteo de Varroa spp}

Los frascos fueron llevados de inmediato al Laboratorio de Inmunología e Investigación de la Facultad de Ciencias Veterinarias de la UNC y se las dejó reposar durante 15 minutos para que las varroas sigan desprendiéndose del cuerpo de las abejas. Luego, empleando los métodos de Montiel y De Jong (Montiel y Piola, 1976; De Jong et al., 1982), las abejas de cada frasco fueron retiradas y contadas. Asimismo, antes de que emerjan completamente de la fase líquida, se las sacudió suavemente tomándolas de una pata para asegurar la caída total de las varroas.

La solución de agua con detergente al $2 \%$ de cada muestra se tamizó en tres oportunidades con un colador de doble malla de $0.87 \mathrm{~mm}$ de luz para retirar los ácaros. Se corroboró la presencia de Varroa atrapados en el colador con una lupa de 10 aumentos. Después, se las separó en un papel toalla para hacer el recuento de ectoparásitos y finalmente fueron trasladados a una placa Petri para su observación empleando un estereoscopio a $20 \mathrm{X}$.

\section{Prevalencia y Análisis Estadístico}

Se calculó la prevalencia en las colmenas, núcleos y de todo el apiario con la fórmula empleada por Arévalo (2018): $P=\# V a$ / \#Ab*100, donde \#Va es el número total de Varroas encontradas en el núcleo, colmena o apiario y \#Ab el número total de abejas muestreadas por núcleo, colmena o apiario. Además, para los análisis estadísticos entre los resultados de Producción y Temperamento se consideró un intervalo de confianza de $95 \%$ y valores menores a 0.05 como estadísticamente significativos con la prueba de Chi cuadrado. El procesamiento de los datos se realizó con el programa Microsoft Excel 2016.

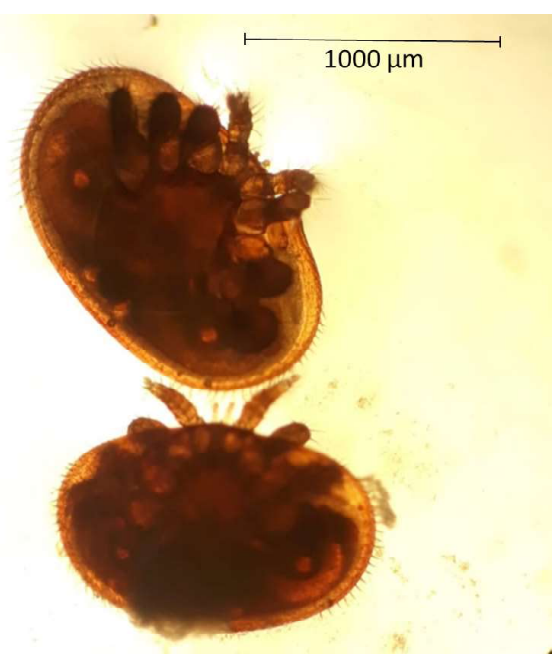

Figura 1. Reconocimiento morfológico de Varroa spp observado en estereoscopio a 20X

\section{Resultados y Discusión}

Los cuatro núcleos $(01-\mathrm{N}, 02-\mathrm{N}, 03-\mathrm{N}$ y $07-\mathrm{N})$ y las tres colmenas $(05-\mathrm{C}, 06-\mathrm{C}$ y $08-C)$ fueron clasificados con buena producción debido a que estaban compuestos por uno o dos pisos. De otra parte, el núcleo 01$\mathrm{N}$ fue clasificado con temperamento «agresivo», el 07-N como «tranquilo», 02-N y 03$\mathrm{N}$ como «muy agresivo», en tanto que las tres colmenas fueron clasificadas de temperamento «muy agresivo».

El núcleo 01-N mostró la mayor prevalencia a varroasis $(3.28 \% \pm 2.20)$, mientras que no hubo presencia del ácaro en las abejas del núcleo $02-\mathrm{N}$. La prevalencia total de los núcleos fue de $1.62 \pm 0.61 \%$ (Cuadro 1 ). Por otro lado, la mayor prevalencia en las colmenas se presentó en la $06-\mathrm{C}$ con $1.66 \%$ \pm 1.1 , siendo la prevalencia total en colmenas de $0.96 \% \pm 0.51$ (Cuadro 2). La prevalencia de Varroa spp total en el apiario fue estimada en $1.39 \% \pm 0.41$. Una vista de los ácaros se presenta en la Figura 1. 
Cuadro 1. Prevalencia de varroasis en los núcleos de abejas del Fundo Tartar Pecuario (Cajamarca, Perú)

\begin{tabular}{ccccccccc}
\hline & \multicolumn{9}{c}{ Núcleo } & \multirow{2}{*}{ Total } & \multirow{2}{*}{ Media } & \multirow{2}{*}{ Varianza } & D.E: \\
\cline { 2 - 7 } & $01-\mathrm{N}$ & $02-\mathrm{N}$ & $03-\mathrm{N}$ & $07-\mathrm{N}$ & & & & \\
\hline Abejas (n) & 305 & 463 & 317 & 580 & 1665 & 416.2 & $17,075.6$ & 130.7 \\
Varroas (n) & 10 & 0 & 2 & 15 & 27 & 6.8 & 48.9 & 7.0 \\
Prevalencia (\%) & 3.28 & 0 & 0.63 & 2.59 & 1.62 & & & \\
I.C. (95\%) & \pm 2.2 & \pm 0 & \pm 0.9 & \pm 1.3 & \pm 0.61 & & & \\
\hline
\end{tabular}

Cuadro 2. Prevalencia de varroasis en las colmenas del Fundo Tartar Pecuario (Cajamarca, Perú)

\begin{tabular}{cccc|cccc}
\hline & \multicolumn{3}{c|}{ Colmenas } & \multirow{2}{*}{ Total } & Media & Varianza & \multirow{2}{*}{ D.E: } \\
\cline { 2 - 4 } & $05-\mathrm{C}$ & $06-\mathrm{C}$ & $08-\mathrm{C}$ & & & & \\
\hline Abejas (n) & 424 & 481 & 531 & 1,436 & 478.7 & $2,866.3$ & 53.5 \\
Varroas (n) & 04 & 08 & 04 & 16 & 5.3 & 5.3 & 2.3 \\
Prevalencia (\%) & 0.94 & 1.66 & 0.75 & 0.96 & & & \\
I.C. (95\%) & \pm 0.9 & \pm 1.1 & \pm 0.7 & \pm 0.51 & & & \\
\hline
\end{tabular}

Se determinó que la cualidad de ser núcleo o colmena no estuvo relacionada con la producción $(\mathrm{p}=0)$ o el temperamento $(\mathrm{p}=0.46667)$. Tampoco influyó en forma significativa con la presencia de Varroa dentro del apiario, lo cual concuerda con las observaciones de Arévalo (2018). Por otro lado, Subía (2013) indica una relación entre el comportamiento y la resistencia de las abejas, asumiendo que las agresivas (africanizadas) son más resistentes a una infestación por Varroa que las abejas tranquilas (europeas); sin embargo dicho estudio no tiene un respaldo estadístico. Por otro lado, Mondet y Conte (2014) manifiestan que la mortalidad de las abejas no está estrechamente relacionada al número de varroas dentro de las colmenas, sino a la capacidad de estas de transmitir enfermedades bacterianas o virales en las colonias. 


\section{Conclusiones}

- A pesar de que las colmenas tienen mayor población de abejas, hubo mayor prevalencia de Varroa spp en los núcleos del apiario.

- El temperamento de los núcleos o colmenas no influyen en la resistencia o susceptibilidad que puedan manifestar ante la varroasis.

- La prevalencia total de Varroa spp en el apiario fue estimada en $1.39 \pm 0.41 \%$.

\section{Literatura Citada}

1. Arechavaleta ME, Guzmán, E. 2000. Producción de miel de colonias de abeja (Apis mellifera L) tratadas y no tratadas con flavulinato contra Varroa jacobsoni Oudemans en Valle de Bravo, Estado de México. Vet México 31: 381-384.

2. Araneda X, Bernales M, Solano J, Mansilla K. 2010. Comportamiento de acicalamiento de abejas (Hymenoptera: Apidae) sobre varroa (Mesostigmata: Varroidae). Rev Colomb Entomol 36: 232-234.

3. Arévalo JL. 2018. Estudio epidemiológico de varroasis en abejas adultas (Apis mellifera) en el Cantón Loja. Tesis de Médico Veterinario Zootecnista. Ecuador: Univ. Nacional de Loja. 56 p.

4. Carreck N, Ball B, Martin S. 2010 Honey bee colony collapse and changes in viral prevalence associated with Varroa destructor. J Apicultural Res 49: 93-94. doi: 10.3896/IBRA.1.49.1.13

5. Dávila M, Ortiz M. 1987. Presencia del ácaro Varroa jacobsoni, ectoparásito de la abeja de la miel en el Perú. Rev Per Entomol 30: 79-80.

6. De Jong D, Roma D, Gonçalves L. 1982. A comparative análisis of shaking solutions for the detection of Varroa jacobsoni on adult honey bees. Apidologie 13: 297-306.
7. Espinoza L. 2004. Varroa destructor. Imagen Vet 4(2): 16-21.

8. Franco C. 2009. Evaluación de tres productos naturales para el control alternativo del ácaro varroa (Varroa destructor) en colmenas de abejas (Apis mellifera) usando gel como sustrato portador. Tesis de Ingeniero Agrónomo. Guatemala: Univ. de San Carlos de Guatemala. $106 \mathrm{p}$.

9. Guerra J, Vieira J, Gonçalves L, De Jong D. 2000. Africanized honey bees (Apis mellifera $\mathrm{L}$ ) are more efficient at removing worker brood artificially infested with the parasitic mite Varroa jacobsoni Oudemans than are Italian bees or Italian/Africanized hybrids. Genet Mol Biol 23: 89-92. doi: 10.1590/ S1415-47572000000100016

10. Locke B, Conte YL, Crauser D, Fries I. 2012. Host adaptations reduce the reproductive success of Varroa destructor in two distinct European honey bee populations. Ecol Evol 2(6): 1144-50. doi: 10.1002/ece3.248. PMID: 22833790; PMCID: PMC3402190.

11. Mantilla J. 2013. Caracterización de enfermedades apícolas (Loque americana, Loque europea, Nosemosis y Varroasis) en el Perú. PRODESA. Servicio Nacional de Sanidad Agraria - Perú [Internet]. Disponible en: https:// www.senasa.-gob.pe/senasa/ descargasarchivos/jer/ABEJAS/INF O R M E \% 2 - 0 F I N A L \% 20 CARACTERI-ZACION\%2-0ENFERMED\%20APICOLAS.pdf

12. Martínez J, Martínez E, Alcalá K, Leal M, Vivas J. 2011. Prevención de varroasis y suplementación. DF México. [Internet]. Disponible en: https:// redgatro.fmvz.unam.mx/assets/ manual_varroosis.pdf

13. Massaccesi C. 2002. Apicultura en la Patagonia andina. Buenos Aires, Argentina. [Internet]. Disponible en: https:// inta.gob.ar/sites/default/files/script-tmpinta-manual_de_apicultura_andina.pdf 
14. Moncada M. 2004. Evaluación del comportamiento de acicalamiento (grooming) de abejas Apis mellifera $\mathrm{L}$ con relación al ácaro Varroa jacobsoni Oud en la comuna de Padres Las Casas, IX Región. Tesis de Ingeniero Agrónomo. Chile: Univ. Católica de Temuco. $73 \mathrm{p}$.

15. Mondet y Conte. 2014. Parasites. In: Titter W (ed). Bee health and Veterinarians. Madrid: España: p 131-141.

16. Montiel E, Piola G. 1976. A new enemy of bees. In: Varroasis a honey bee disease. Bucharest, Romania: Apimondia Publishing House. p 36-37.

\section{Panziera $D$, Langevelde $F$, Blacquière} T. 2017. Varroa sensitive hygiene contributes to naturally selected varroa resistance in honey bees. J Apicultural Res 56: 635-642. doi: 10.1080/ 00218839.2017 .1351860

18. Polaino C. 2006. Manual práctico del apicultor. Madrid, España. p 381-387.

19. Subía S. 2013. Determinación de la prevalencia de varroa (Varroa destructor), y posibles factores de riesgo, en dos apiarios ubicados en la provincia de Pichincha-Ecuador. Tesis de Médico Veterinario Zootecnista. Ecuador: Univ. de las Américas. 48 p. 